\title{
Planung, Vorbereitung und Kontrolle bei Eingriffen in der Veterinärmedizin
}

\author{
Andrea Meyer-Lindenberg
}

\section{Zusammenfassung}

Die Ruptur des vorderen Kreuzbands ist beim Hund eine der häufigsten Lahmheitsursachen der Hintergliedmaße. Im Gegensatz zum Menschen entsteht die Ruptur des vorderen Kreuzbands beim Hund häufiger durch degenerative Veränderungen des Bandes. Für die Therapie steht eine große Vielzahl von Operationstechniken zur Verfügung, wobei sich insbesondere bei großwüchsigen Hunderassen Verfahren bewährt haben, bei denen durch verschiedene chirurgische Techniken Kniegelenksstatik und -biomechanik beeinflusst werden. Eines dieser Operationsverfahren ist die Tibia-PlateauLevelling-Osteotomie (TPLO). Hierbei werden die Gelenkanatomie, die Muskelfunktion und das Körpergewicht als ein zusammenarbeitendes System betrachtet mit dem Ziel, die Funktion des kranialen Kreuzbands zu neutralisieren. Auf einen direkten Ersatz des vorderen Kreuzbands kann daher verzichtet werden. Durch die Anhebung des nach kaudal abschüssigen Tibiaplateaus wird hierbei mittels Korrekturosteotomie die kraniale Translation der
Tibia aufgehoben und in einen Gravitationsschub umgewandelt. Dieser Schritt wird durch das Anlegen eines halbkreisförmigen Sägeschnitts in einem individuell zu bestimmenden Bereich der proximalen Tibia erreicht, wodurch das Tibiaplateau rotiert und nach kaudal angehoben werden kann. Durch die Rotation des Tibiaplateaus auf einen Winkel von etwa $6,5^{\circ}$ wird der kraniale Schub aufgehoben und das kaudale Kreuzband übernimmt zusätzlich die stabilisierende Komponente im Kniegelenk. Das Operationsverfahren der TPLO wird in der vorliegenden Arbeit detailliert beschrieben.

Planning, Preparation and Control of Interventions in Veterinary Medicine

Rupture of the anterior cruciate ligament is the most frequent cause of hind leg paralysis in dogs. In contrast to humans, rupture of the anterior cruciate ligament in dogs is more often due to degenerative changes of the ligament. Numerous surgical techniques are available for treatment and, especially for the larger dog breeds, those methods have proved to be helpful in which the stability and biomechanics of the joint are influenced by various surgical techniques. One of these operative procedures is the tibia plateau levelling osteotomy (TPLO). Here, the joint anatomy, muscle function and body weight are considered as a mutually interacting system with the aim to neutralise the function of the cranial cruciate ligament. In this way a direct replacement of the anterior cruciate ligament can be avoided. By elevating the caudally down-sloping tibial plateau the cranial translation of the tibia is given up and transformed into a gravitational push by means of an appropriate correction osteotomy. This step is achieved by making a semicircular saw cut in an individually predetermined region of the proximal tibia whereby the tibial plateau can be rotated and elevated caudally. By rotation of the tibial plateau to an angle of about $6.5^{\circ}$, the cranial force is eliminated and the caudal cruciate ligament additionally takes on the function of a stabilising component in the knee joint. The operative procedure of TPLO is described in detail in the present article.

\section{Einleitung}

Die Veterinärmedizin unterlag in den letzten Jahrzehnten einem großen Wandel. Während früher die Diagnostik und Therapie von Erkrankungen von Nutztieren im Zentrum standen, rückt seit Mitte des 20. Jahrhunderts die Versorgung von Haustieren, insbesondere von Hunden und Katzen, in den Vordergrund. Parallel zu der Entwicklung in der Humanmedizin stiegen die Ansprüche des Tier-

OP-JOURNAL 2011; 27: 90-98

(c) Georg Thieme Verlag KG Stuttgart · New York DOI http://dx.doi.org/10.1055/s-0030-1271016 besitzers an den Tierarzt. Dies führte zur Expansion der diagnostischen und therapeutischen Verfahren, die sich nach und nach immer mehr in der Veterinärmedizin etabliert haben.

Damit fanden auch die unterschiedlichsten Operationstechniken den Einzug in die veterinärmedizinische Weichteilund Knochenchirurgie. In Bezug auf eine spezielle Planung und Vorbereitung eines operativen Eingriffs stehen insbesondere verschiedene Erkrankungen des Bewegungsapparats im Vordergrund, wobei im Folgenden auf einen ausgewählten orthopädischen Eingriff am Kniegelenk (Tibia-Plateau-Levelling-Os- teotomie) eingegangen werden soll. Zum besseren Verständnis der Planung des operativen Eingriffs werden einige Grundlagen zur Anatomie, Entstehung und Diagnose vorangestellt.

\section{Das Kniegelenk}

\section{Anatomische Gesichtspunkte}

Das Kniegelenk des Hundes ist ähnlich dem des Menschen aufgebaut.

Das distale Endstück des Femurs besteht aus dem kaudodistal abgebogenen Condylus medialis und dem Condylus lateralis. Das proximale Endstück der Tibia 


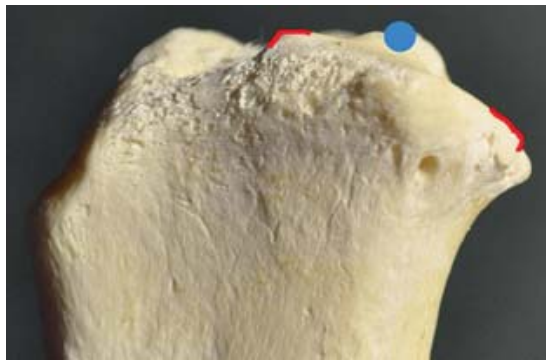

Abb. 1 Darstellung der proximalen Tibiagelenkfläche in seitlicher Ansicht mit rot gekennzeichneter kranialer und kaudaler Begrenzung der medialen Gelenkfläche der proximalen Tibia und dem Mittelpunkt der Tuberculi intercondylare mediale und laterale (blauer Kreis).

wird ebenfalls aus 2 Gelenkflächen gebildet, dem Condylus lateralis und dem Condylus medialis. Zwischen den beiden Kondylen erhebt sich die knorpelfreie Eminentia intercondylaris, an der, durch die Area intercondylaris centralis getrennt, das Tuberculum intercondylare laterale und mediale ausgebildet sind (Abb.1). Die kraniale Begrenzung stellt die Tuberositas tibiae dar, eine kräftig ausgebildete Knochenleiste als Ansatzstelle für den M. quadriceps femoris.

Neben den beiden Kollateralbändern und dem aus dem M. quadriceps femoris hervorgehenden und an der Tub. tibae ansetzenden Lig. patellae mit der eingelagerten Patella besitzt der Hund 2 Menisken, die die Inkongruenz im Kniegelenk ausgleichen. Ebenfalls wie der Mensch hat der Hund 2 Kreuzbänder: das kraniale und das kaudale Kreuzband. Die wichtigste Funktion beider Kreuzbänder hat beim Hund das kraniale Kreuzband, weshalb es im Falle einer Schädigung schwerwiegende Folgen hat. Das intakte kraniale Kreuzband begrenzt die Verschieblichkeit der Tibia nach vorne, aber auch die Innenrotation der Tibia sowie die Hyperextension des Kniegelenks [1].

Eine weitere wichtige Rolle spielt die Muskelkoaktivität. Darunter versteht man die Funktion der Muskulatur zur Erhaltung der Kniegelenkstabilität. Man kann die Muskelgruppen im Hinblick auf die Aufgabe des vorderen Kreuzbands in Agonisten bzw. Antagonisten unterteilen $[1,2]$. Die Muskulatur (M. biceps femoris, M. semimembranosus, M. semitendinosus, M. abductor cruris caudalis, M. gracilis, M. sartorius) unterstützt die Funktion des vorderen Kreuzbands, indem sie eine auf die proximale Tibia nach kaudal gerichtete Zugwirkung ausübt und somit als Agonist des kranialen

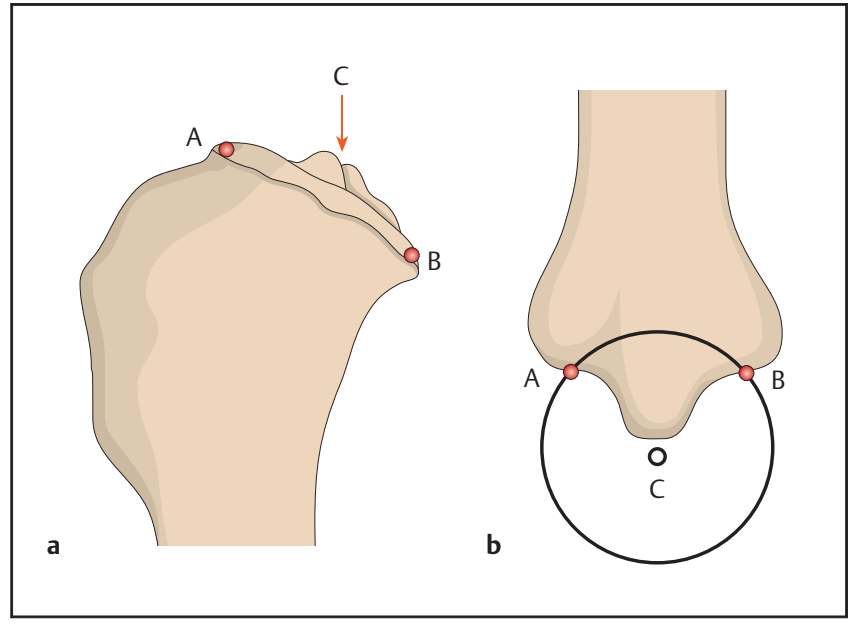

Abb. $2 \mathrm{a}$ und $\mathrm{b}$

Schematische Darstellung der Bestimmung der mechanischen Achse der Tibia. (a) A und $B$ kennzeichnen den kranialsten und kaudalsten Punkt des Tibiaplateaus, der Pfeil kennzeichnet mit C den Punkt zwischen den beiden Tuberculi intercondylare. (b) A und $B$ kennzeichnen die kraniale und kaudale Begrenzung der Tibialgelenkfläche und Punkt C stellt das Zentrum des Os tali dar.
Kreuzbands arbeitet. Der M. quadriceps ist dagegen ein biomechanischer Antagonist und die einzige Muskelgruppe, die mit kranioproximalem Zug auf die Tibia einwirkt. Diese Zugkraft wird durch die Patella umgeleitet und teilt sich in eine Zugwirkung auf die Tibia nach kranioproximal und eine Druckwirkung auf die Femurkondylen nach kaudal. Der M. gastrocnemius fungiert ebenfalls als Antagonist, indem er eine starke kaudale Zugkraft auf den distalen Femur erzeugt.

Die Hauptbelastung während der Standund Belastungsphase trägt das Tibiaplateau, wobei es sich beim Tibiaplateau um die den Femurkondylen gegenüberliegenden medialen und lateralen Gelenkflächen der proximalen Tibia handelt. Die topografische Anatomie des Tibiaplateaus des Hundes zeigt in der transversalen Ebene konkav erscheinende Kondylen und in der sagittalen Ebene konvex erscheinende Kondylen. Aus der Seitenansicht ergibt sich eine Neigung der Tibiakondylen nach kaudodistal [3].

Durch die Neigung des Tibiaplateaus lastet bei jedem Schritt ein Vorwärtsschub (cranial tibial thrust - CCT) auf den Kreuzbändern, insbesondere dem kranialen Kreuzband.

Je steiler nun die Gelenkfläche steht, umso größer ist dieser Vorwärtsschub. Dieses Phänomen kann mit einer schiefen Ebene verglichen werden.

\section{Messungen zur Erkennung von Abweichungen des Tibiaplateauwinkels in der seitlichen Ebene}

Der Neigungswinkel des Tibiaplateaus wird mithilfe von speziellen Messpunkten bestimmt. Auf einem gut gelagerten

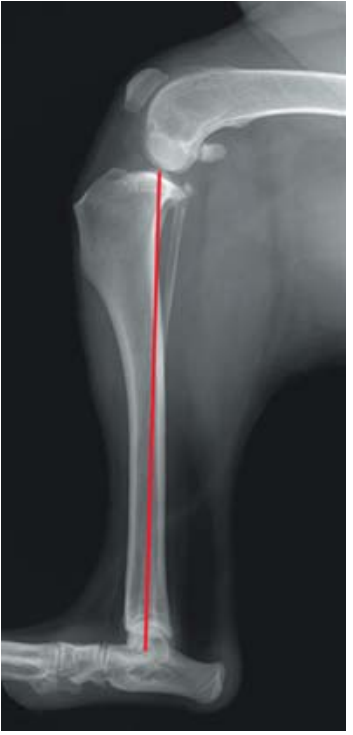

Abb. 3 Darstellung der mechanischen Achse (schwarze Linie) der Tibia am mediolateralen Röntgenbild.

mediolateralen Röntgenbild wird zunächst die Tibiaachse durch eine Gerade bestimmt. Die Gerade verläuft distal vom Zentrum des Os tali (Abb.2b) zum höchsten Punkt der Tibia, zwischen den Tuberculi intercondylare (Abb. 2a, 3).

Das Tibiaplateau selbst wird durch eine Gerade gebildet, die der medialen Gelenkfläche der proximalen Tibia entspricht. Deren Begrenzung ist kranial eine kleine Erhebung an der medialen Gelenkfläche, die dem Ansatz des vorderen Kreuzbands entspricht, und kaudal ist es die kaudale Begrenzung der Gelenkfläche (Abb.4). Der Tibial Plateau Slope (TPS) bezeichnet den Winkel zwischen der Tibiaachse und dem Tibiaplateau. 


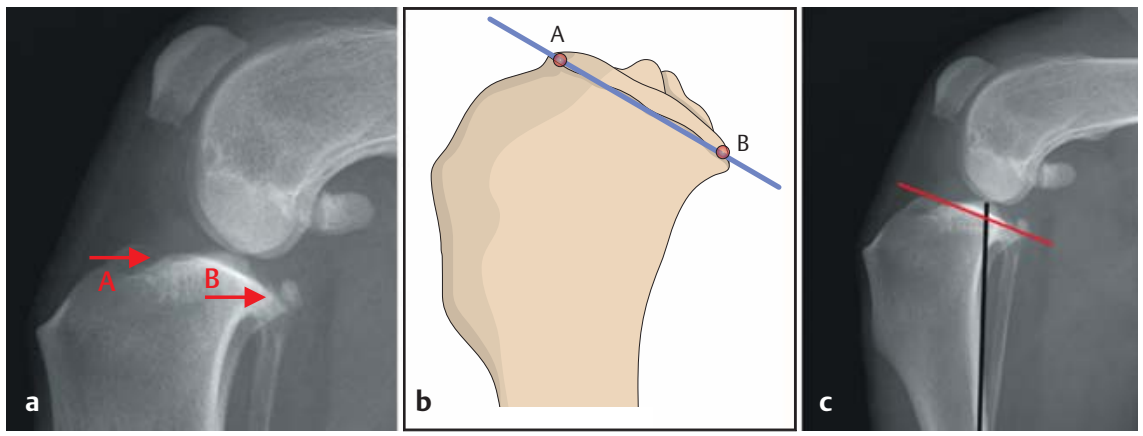

Abb. 4a bis c (a) Bestimmung der Punkte der kranialen (A) und kaudalen (B) Tibiaplateaugrenze am Röntgenbild. (b) Schematische Darstellung der beiden Punkte des Plateaus mit Ziehung einer Geraden durch das Tibiaplateau. (c) Röntgenbild im mediolateralen Strahlengang mit Darstellung der mechanischen Tibiaachse und des Tibiaplateaus.
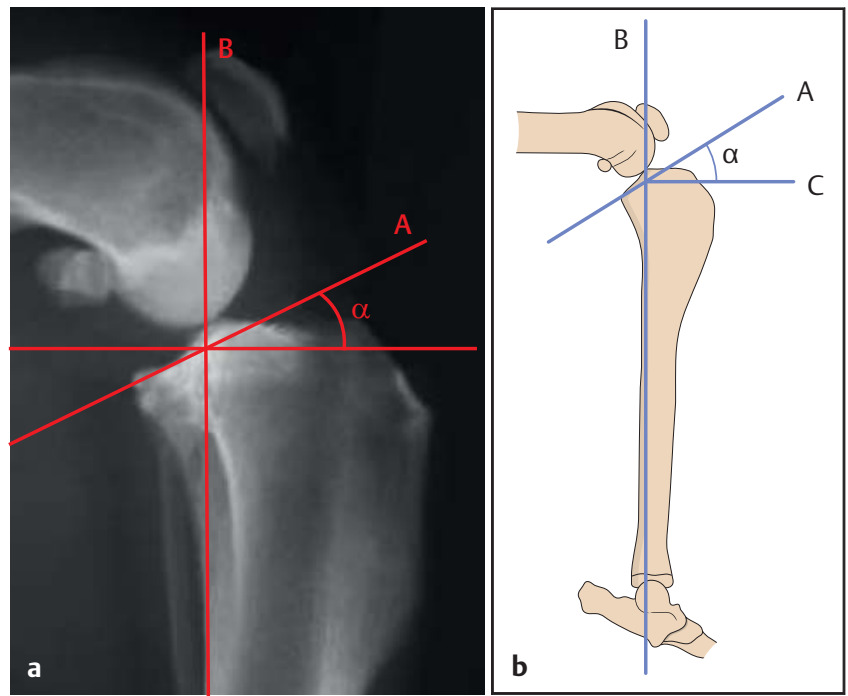

Abb. 5 a und $b$

(a) Bestimmung des TPA am Röntgenbild des linken Kniegelenks: B - mechanische Tibiaachse, A - Linie des Tibiaplateaus, Tibiaplateauwinkel $(\alpha)$. (b) Schematische Darstellung an der Zeichnung einer ganzen Tibia: $B$ - mechanische Tibiaachse, A - Linie des Tibiaplateaus, C - Senkrechte zur mechanischen Tibiaachse mit dem entsprechenden Tibiaplateauwinkel $(\alpha)$.

Der Winkel zwischen einer gebildeten Senkrechten zur Tibiaachse und der Geraden, die das Tibiaplateau bildet (Abb.5), wird als der Neigungswinkel des Tibiaplateaus bezeichnet (Tibia-Plateau-Angle - TPA).

Zur Erhebung konstanter Ergebnisse sind standardisierte Röntgenaufnahmen mit korrekter Lagerung zur Messung des TPA sehr wichtig.

Eine fehlerhafte Röntgentechnik kann die radiologische Erscheinung des TPA sehr stark verändern, sodass es zu Fehlmessungen der Winkelung kommt. Diese können den Erfolg einer geplanten Operation gefährden. Der TPA ist bei den verschiedenen Hunderassen unterschiedlich, wie auch die Winkelung der Kniegelenke im Stand bei verschiedenen Rassen unterschiedlich ist. Der TPA liegt bei gesunden Hunden der meisten Rassen unter $20^{\circ}$ [4].
Femurkortizes geteilt, beide interkondylaren Eminentiae müssen erkennbar sein und die mediale Kante des Kalkaneus sollte über dem tiefsten Punkt des Sulcus tali liegen (Abb.6). Es werden Messpunkte für den mechanischen medialen proximalen (mMPTA) und den distalen (mMDTA) Tibiawinkel in der Frontalebene (Abb. 6) festgelegt [6]. Der errechnete mittlere mMPTA (medial mechanical proximal tibia angle) liegt bei ca. $90^{\circ}$ und der mMDTA (distal medial mechanical tibia angle) bei etwa $93^{\circ}$. Es werden keine signifikanten Unterschiede bei gesunden Gliedmaßen zwischen den verschiedenen Hunderassen beschrieben.

\section{Ruptur des kranialen Kreuzbands}

Eine beim Hund sehr häufig vorkommende orthopädische Erkrankung der Hintergliedmaße ist die Ruptur des kranialen Kreuzbands (Lig. cruciatum craniale), die in der Regel mit einer deutlichen Lahmheit verbunden ist.

\section{Vorkommen, Ätiologie und Pathogenese}

Im Gegensatz zum Menschen, bei dem die Ursache für einen Kreuzbandriss häufig traumatisch bedingt ist, kommt es beim Hund häufiger durch degenerative Veränderungen des Bandes zu einer Ruptur [7].

Die Ursache der degenerativen Veränderungen ist multifaktoriell. Neben rezidivierenden Entzündungen, immunologischen Prozessen, Alterungsprozessen oder Übergewicht kommen auch Instabilitäten (z.B. Patellaluxation) und Fehlstellungen (Valgusstellung, Varusstellung) der Hintergliedmaße als auslösende Faktoren infrage. Weiterhin wird diskutiert, dass eine steile Winkelung/Neigung des Tibiaplateaus (tibial slope) als anatomischer Faktor prädisponierend für Kreuzbandrupturen sein könnte [8]. Sowohl großwüchsige als auch kleine Rassen können betroffen sein, wobei die großen Rassen häufiger bis zum 5. Lebensjahr erkranken, die kleinen hingegen oft über 7 Jahre alt sind. Eine Rassenprädisposition ist nicht gesichert, obwohl manche Rassen häufiger an einem Kreuzbandriss erkranken.

Bei einer Teilruptur oder Ruptur des kranialen Kreuzbands verliert das Kniegelenk immer mehr an Stabilität und es kommt ohne operativen Eingriff $\mathrm{zu}$ sekundären Veränderungen, wie die Entstehung von Arthrosen, einer Gelenk- 


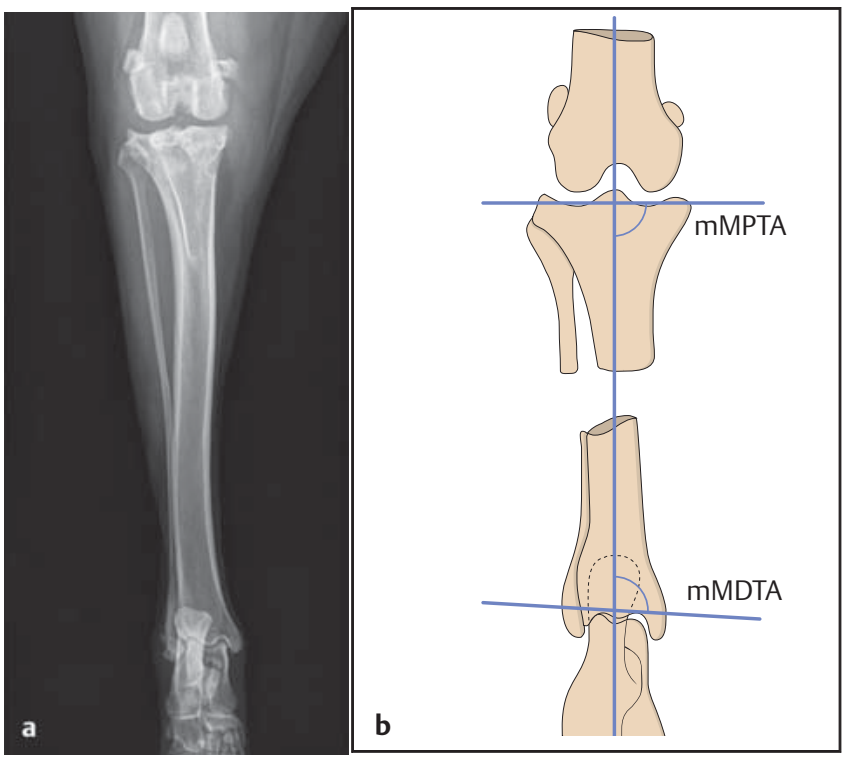

kapselfibrose und von Gelenkknorpelschäden.

Weiterhin ist der Kreuzbandriss meist mit einer sekundären Meniskusschädigung des kaudalen Anteils des inneren Meniskus vergesellschaftet [1]. Nur sehr selten werden beim Hund primäre Meniskusläsionen beobachtet.

\section{Diagnose des vorderen Kreuzbandrisses}

Die Diagnose eines vorderen Kreuzbandrisses wird anhand der Anamnese, der klinischen Symptome und des Palpationsbefunds gestellt. Klinisch zeigt der Hund eine gering- bis hochgradige Lahmheit der Hintergliedmaße mit Entlastungshaltung und Zehenspitzenstellung im Stand. Palpatorisch ist das Kniegelenk oft verdickt und weist anhand des Schubladenphänomens, bei der sich die Tibia in Relation zum Femur nach kranial verschieben lässt, eine mehr oder weniger deutliche Instabilität auf. Eine weitere Möglichkeit zur Diagnose bietet der Tibiakompressionstest (Abb. 7). Während der Stützphase wird die Tibia zwischen Femur und Tarsus „komprimiert“. Dies geschieht zum einen durch das Körpergewicht und zum anderen durch die Kontraktion des M. gastrocnemius, der die Tibia vom Femur bis zum Kalkaneus überspannt. Diese durch den Tibiakompressionsmechanismus erzeugten Kräfte wirken antagonistisch zur Funktion des vorderen Kreuzbands [2].

Der Tibiakompressionstest wird bei einem Beugewinkel des Kniegelenks von etwa $140^{\circ}$ durchgeführt. Mit der einen Hand wird der distale Femur umgriffen,
Abb. $6 \mathrm{a}$ und $\mathrm{b}$

Schematische Darstellung der Winkelmessung zwischen der mechanischen Achse und den Gelenkorientierungslinien: proximaler oberer Winkel, mMPTA (mechanischer medialer proximaler Tibiawinkel in der Frontalebene); distaler unterer Winkel, mMDTA (mechanischer medialer distaler Tibiawinkel der Frontalebene).

wobei der Zeigefinger auf der Tuberositas tibiae liegt und diese leicht nach kaudal presst. Mit der anderen Hand wird das Tarsalgelenk alternierend gebeugt und gestreckt. Die Kraft und die Kontraktion des M. gastrocnemius führen bei Beugung des Tarsalgelenks dazu (Abb. 7), dass die proximale Tibia nach kranial gleitet (subluxiert).

Röntgenaufnahmen sollten zur Diagnose und Planung der Therapie immer in 2 Ebenen angefertigt werden. Für die Diagnose dient die Erkennung von Sekundärveränderungen wie vermehrte Gelenksfüllung oder Arthrosen. In unklaren Fällen oder zur Erkennung von Meniskusläsionen kann eine Magnetresonanztomografie vorgenommen werden. Neben der meist vermehrten Gelenksfüllung und dem rupturierten Kreuzband können mit diesem Verfahren Meniskus- und Knorpelläsionen oder Veränderungen im Knochen (z.B. Ödeme) oder umgebenden Weichgewebe beurteilt werden. Zur Erkennung von sekundären Meniskusläsionen kann auch die Arthroskopie herangezogen werden.

\section{Therapiemöglichkeiten bei der vorderen Kreuzbandruptur}

Zur Therapie der vorderen Kreuzbandruptur wurden zahlreiche Operationsverfahren und Modifikationen beschrieben. Sie können grundsätzlich in solche unterteilt werden, die die Gelenkstatik verändern und solche, die sie nicht verändern. Letztere können in extrakapsuläre und intrakapsuläre Bandersatzmethoden unterschieden werden.

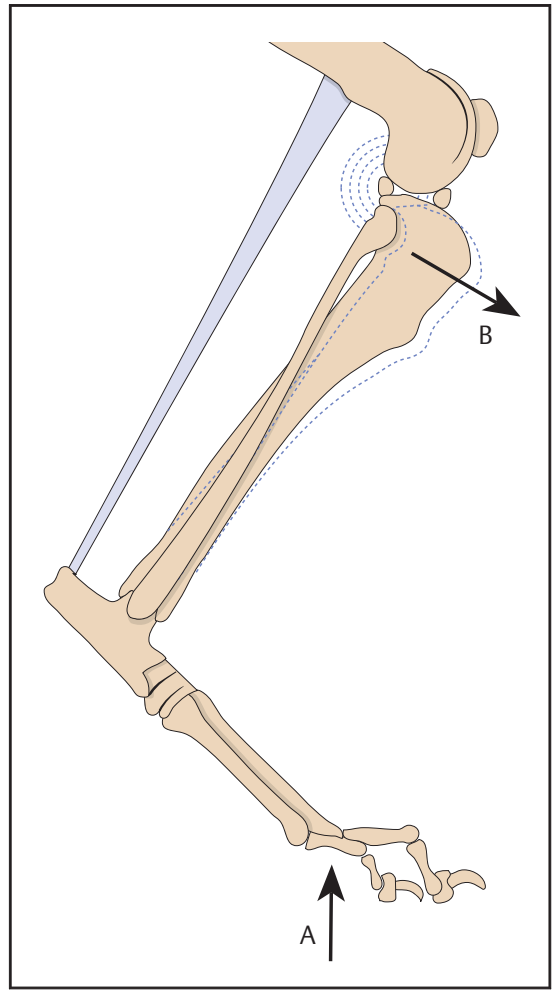

Abb. 7 Schematische Darstellung des Tibiakompressionstests: Bei Beugung des Tarsalgelenks durch Druck auf die Pfote (A) kommt es bei in $140^{\circ}$ fixiertem Knie zu einer Kranialverschiebung der proximalen Tibia (B).

Besonders bei mittelgroßen und großen Hunden über $20 \mathrm{~kg}$ Körpermasse ist aufgrund der starken auf das Gelenk einwirkenden Kräfte eine chirurgische Versorgung des rupturierten Bandes mit herkömmlichen Methoden, die die Gelenkstatik nicht verändern (z.B. künstlicher Bandersatz, Kapselfaszienraffung) nicht immer erfolgreich, da das eingebrachte Bandmaterial reißen kann oder es im Laufe der Heilung zu einer erneuten Instabilität durch Lockerung der Stabilisierungsmethode mit entsprechenden Folgen, wie erneute Lahmheit, Fortscheiten der Arthrosen oder Meniskusschäden, kommen kann [9].

Daher wurden zur Therapie einer Ruptur des kranialen Kreuzbands insbesondere für die mittel bis- großwüchsigen Rassen verschiedene Operationsmethoden entwickelt, die zu einer Veränderung der Gelenkstatik führen.

Dabei werden die Gelenkanatomie, die Muskelfunktion und das Körpergewicht als ein zusammenarbeitendes System betrachtet mit dem Ziel, die Funktion des kranialen Kreuzbands zu neutralisieren. Dadurch kann auf einen direkten Ersatz des vorderen Kreuzbands verzichtet 


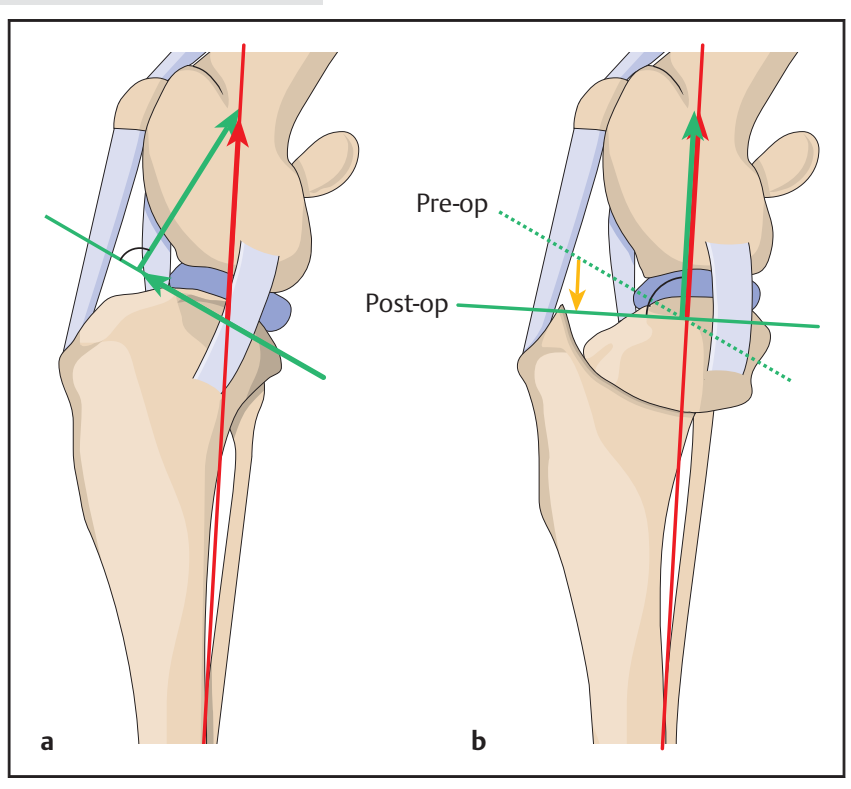

werden. Die Kräfte, die auf das Tibiaplateau wirken, werden zum kaudalen Kreuzband geleitet. Durch eine Umstellungsosteotomie soll eine muskuläre Kompensation des CTT während der Belastungsphase der Gliedmaße ermöglicht werden.

Eines dieser Operationsverfahren ist die Tibia-Plateau-Levelling-Osteotomie (TPLO), deren Planung, Vorbereitung und Kontrolle nachfolgend näher beschrieben werden soll.

\section{Prinzipien der TPLO}

Während der Gewichtsbelastung der Hintergliedmaße ist die axiale Reaktionskraft entlang der Längsachse der Tibia ausgerichtet. Bei Auftreffen auf das von kranial nach kaudal abfallende Tibiaplateau wird diese Kraft in eine Kompressionskraft umgewandelt (Vektor senkrecht zum Tibiaplateau) und in eine nach kranial gerichtete Kraft (Vektor parallel zum Tibiaplateau), durch die die Kranialverlagerung der Tibia ausgelöst wird. Nach Ruptur des kranialen Kreuzbands verursacht die Gewichtsbelastung also eine Kranialverschiebung der Tibia $[10,11]$.

Ziel der die Gelenkstatik und Biomechanik des Kniegelenks verändernde Operationsmethode der TPLO ist es, durch die Anhebung des nach kaudal abschüssigen Tibiaplateaus mittels Korrekturosteotomie die kraniale Translation der Tibia (CTT) aufzuheben und diese in einen Gravitationsschub umzuwandeln [12].
Abb. 8 a und $b$

Nach Durchführung der Osteotomie kann der CTT durch Rotation des Tibiaplateaus auf ca. $6,5^{\circ}$ ausgeschaltet werden. Es kommt zu einer nach unten gerichteten Schubkraft.

Dieser Schritt wird durch das Anlegen eines halbkreisförmigen Sägeschnitts in einem individuell zu bestimmenden Bereich der proximalen Tibia erreicht, wodurch das Tibiaplateau rotiert und nach kaudal angehoben werden kann. Damit wird die Biomechanik des Kniegelenks dahingehend geändert, dass es während der Standphase (Gewichtsbelastung) zu einer funktionellen Stabilität des Gelenks kommt. Durch die Rotation des Tibiaplateaus auf einen Winkel von etwa $6,5^{\circ}( \pm 0,9)$ wird der kraniale Schub aufgehoben und das kaudale Kreuzband übernimmt zusätzlich die stabilisierende Komponente im Kniegelenk [9,13].

Die Schubladenbewegung wird zudem muskulär kompensiert. Das heißt, dass nur der CTT, nicht aber das Schubladenphänomen eliminiert wird [12]. Das Vorhandensein des hinteren Kreuzbands ist dabei eine Grundvoraussetzung für die Auswahl und Durchführung des Verfahrens, da es nach Durchführung der TPLO zu einer Zunahme der Kräfte am hinteren Kreuzband kommt [14]. Eine Überschreitung der Winkelung von $6,5^{\circ}$ führt zu einer zunehmenden Belastung des hinteren Kreuzbands mit der Gefahr der Schädigung des Bandes $[9,13]$. Theoretisch wird die größte Effektivität bei einer TPLO erzielt, wenn dieser minimale Tibiaplateauwinkel erreicht wird [10]. Das Drehen des Plateaus wird durch eine zirkuläre Osteotomie ermöglicht (Abb. 8). Nach der Osteotomie kann das Tibiaplateau rotiert und in dem idealen Winkel mit einer Platte fixiert werden.
Planung am Röntgenbild und Ermittlung der erforderlichen Rotation des Tibiaplateaus

Es werden 2 Röntgenbilder im mediolateralen und kraniokaudalen Strahlengang angefertigt. An der mediolateralen Aufnahme wird der Rotationsgrad des Tibiaplateaus bestimmt und auf der kraniokaudalen ggf. eine erforderliche Achsenkorrektur.

Auf dem gut gelagerten Röntgenbild im mediolateralen Strahlengang (Kniegelenk und Tarsalgelenk mit abgebildet, jeweils im Winkel von $90^{\circ}$, Femurkondylen deckungsgleich, Fibula im proximalen Drittel deutlich von der Tibia abgesetzt) wird zunächst die mechanische Längsachse der Tibia bestimmt, die Tibiaplateaulinie markiert und auf dem Röntgenbild aufgetragen. Anschließend wird im Schnittpunkt der beiden Geraden eine Senkrechte zur Tibialängsachse angelegt und der Tibiaplateauwinkel bestimmt (Abb. 9).

Anschließend muss anhand dieses Röntgenbilds die Position der Osteotomie und die Größe des zu verwendenden Sägeblatts ermittelt werden. Die ideale Osteotomie sollte auf dem höchsten Punkt der Längsachse (Eminentiae) zentriert sein, da dies mathematisch der optimale Drehpunkt des Kniegelenks (instant center of rotation - ICR) ist.

Die Ermittlung der Osteotomieposition erfolgt anhand einer vorgefertigten Schablone, auf der die verschiedenen Größen der Sägeblätter aufgetragen sind. Es ist bei der Wahl des Sägeblatts und der Schnittführung zu berücksichtigen, dass eine ausreichende Breite der Tub. tibiae stehen bleibt (Abb. 10).

Mindestens $1 \mathrm{~cm}$ sollte der Abstand zum Ansatz des Lig. patellae betragen. Weiterhin sollte die Größe des proximalen Fragments zur Fixation der Platte ausreichend sein und der Schnitt sollte keine Verletzung des Gelenkknorpels der Tibia, des Lig. patellae oder des Bandes zwischen den Menisken hervorrufen.

Ist die Größe des Sägeblatts bestimmt, müssen am Röntgenbild 2 Distanzen (D1 und D2) bestimmt werden, um die korrekte Lage des Sägeschnitts in der späteren Operation auf den Knochen übertragen zu können. Dafür wird vom Ansatz des Lig. patellae (Referenzpunkt) zunächst die Distanz zum proximalsten Osteotomiepunkt bestimmt (D1) und 


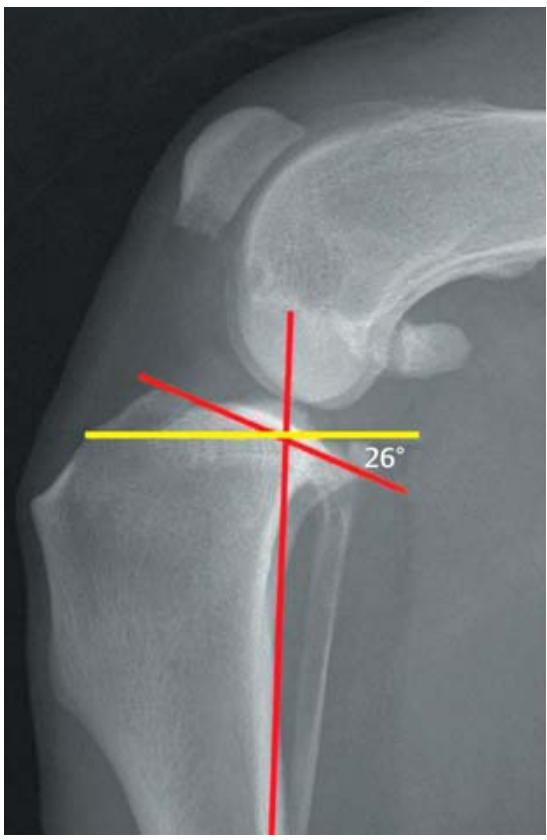

Abb. 9 Bestimmung des Tibiaplateauwinkels am Röntgenbild, er beträgt hier $26^{\circ}$.

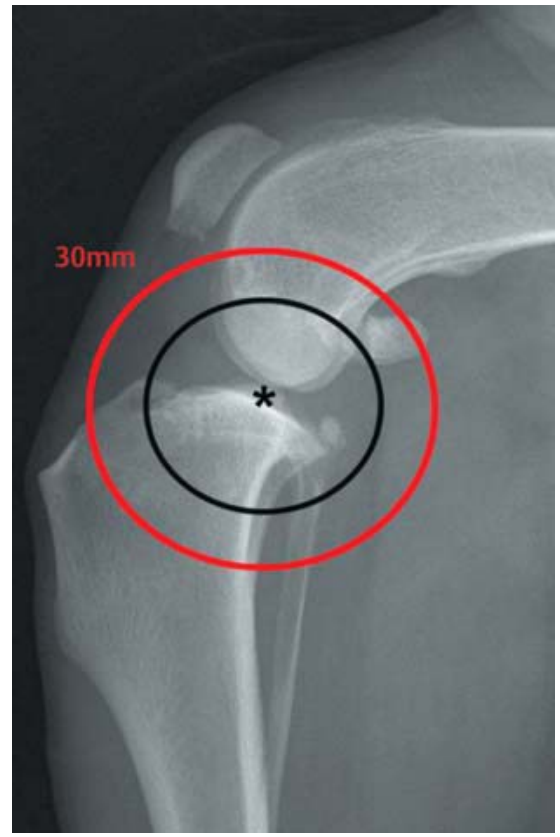

Abb. 10 Auswahl des Sägeblattdiameters (rot: $30 \mathrm{~mm}$ - korrekte Größe, schwarz: $24 \mathrm{~mm}$ ) mit Zentrum im ICR $\left({ }^{*}\right)$, mit dem eine ausreichende Breite der Tub. tibiae stehenbleibt.
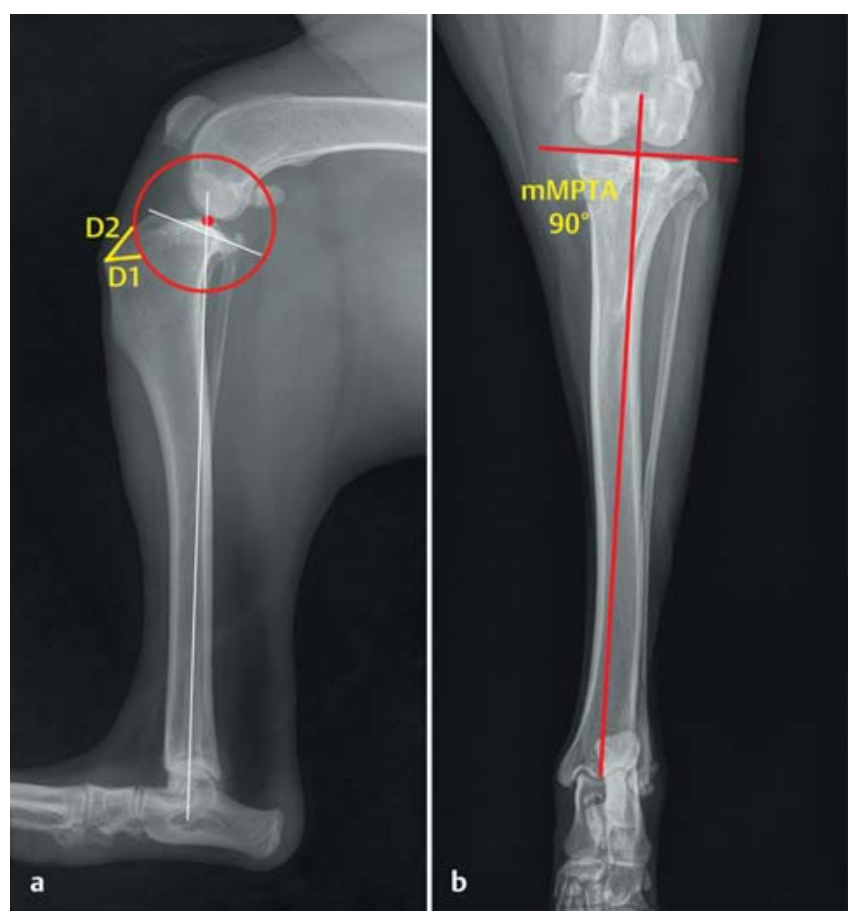

Abb. 11 a und $b$ Präoperative Planung der Rotation des Tibiaplateaus am mediolateralen Röntgenbild (a) und zur Achsenkorrektur am kraniokaudalen Röntgenbild (b) bei Durchführung einer TPLO zur Therapie einer Kreuzbandruptur.

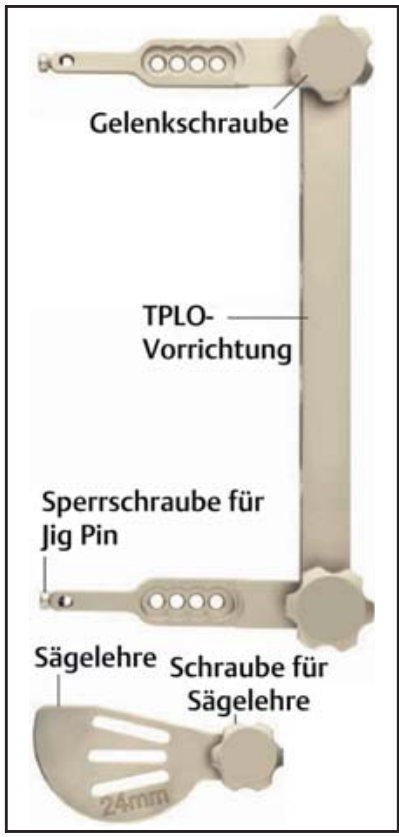

Abb. 12 TPLO-Jig der Fa. Synthes, der über 2 Kirschner-Bohrdrähte in spezieller Weise an der Tibia fixiert wird. Die Sägelehre (hier für ein $24 \mathrm{~mm}$-Sägeblatt) wird nach Anpassen in der dafür vorgesehenen Vorrichtung fixiert.

mechanische Tibiawinkel (mPMTA normal $90^{\circ}$ ) vergrößert bzw. verkleinert sind, sollte $\mathrm{ab}$ einer Abweichung der Achse von $10^{\circ}$ korrigiert werden. Nach Festlegung der entsprechenden Sägeblattgröße und Bestimmung des Winkels des Tibiaplateaus wird die erforderliche Rotation des Tibiaplateaus anhand einer Tabelle (Tab. 1) in Millimeter abgelesen.

\section{Vorbereitung des Patienten und Kontrolle des Eingriffs}

Für den operativen Eingriff sind neben einem Grundinstrumentarium und Instrumenten für die Knochenchirurgie spezielle Instrumente für die TPLO (spezielle Sägeblätter, TPLO-Jig) und Spezialplatten für die Fixation des Tibiaplateaus erforderlich. Bezüglich der Platten gibt es inzwischen eine Vielzahl an unterschiedlichen Modellen, wobei sich insbesondere winkelstabile Plattensysteme (z. B. Fa. Synthes [Umkirch], Fa. Orthomed [Kaltenkirchen]) bewährt haben.

Der operative Eingriff erfolgt in Allgemeinanästhesie. Nach chirurgischer Vorbereitung der betroffenen Gliedmaße erfolgt die Lagerung des Patienten in Seitenlage oder in Rückenlage. Ggf. nach vorheriger Arthroskopie zur Diagnostik und Therapie von Meniskusläsionen oder zur Durchführung eines Meniskusrelease erfolgt der Zugang zum Kniegelenk und 


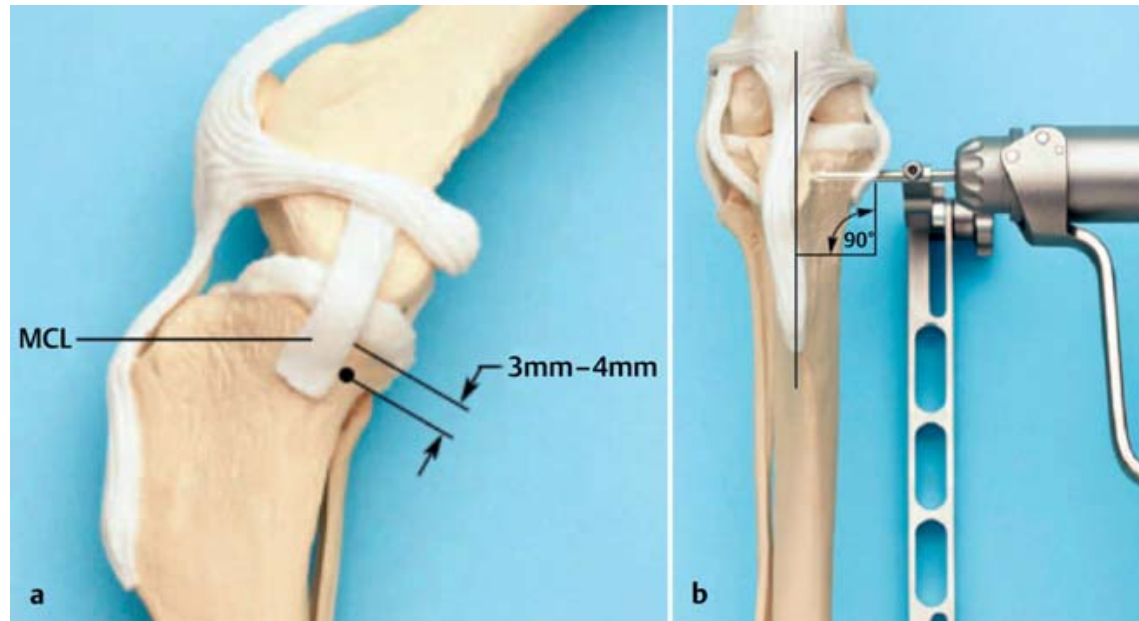

Abb. 13a und b Die Position des TPLO-jigs erfolgt direkt kaudal des medialen Seitenbands (MCL) etwa 3-4 mm unterhalb der Gelenkfläche (a), in einem Winkel von $90^{\circ}$ zur Achse der Tibia (b).

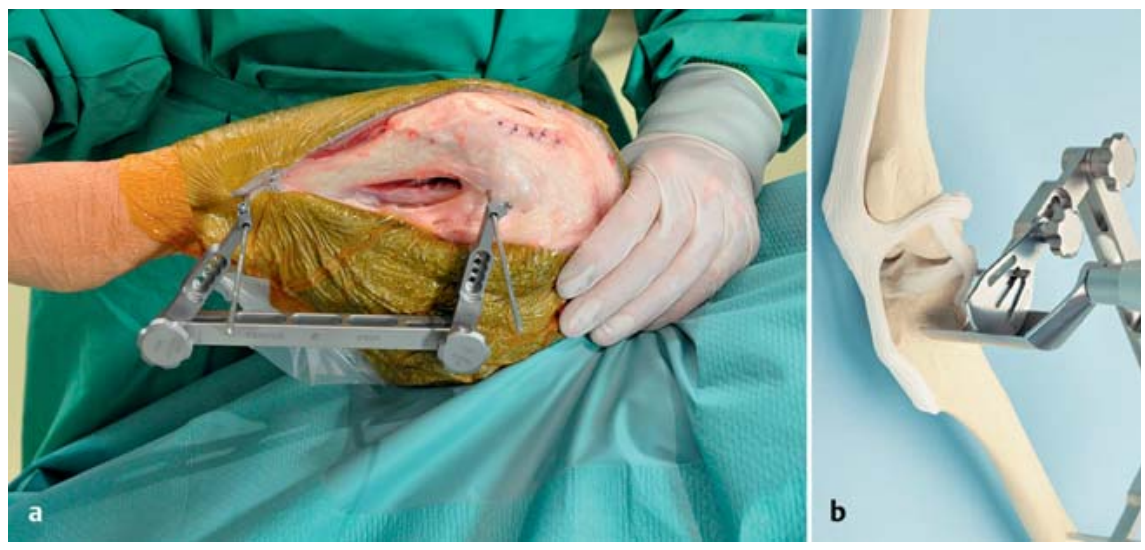

Abb. 14a und $\mathbf{b}$ Operationssitus mit Positionierung des TPLO-jigs an der Tibia. Die Fixierung erfolgt an den beiden in die Tibia gebohrten proximalen und distalen Kirschner-Bohrdrähten (a). Anschließend wird die Sägelehre an dem Jig fixiert, um dem gewählten Sägeblatt eine sichere Führung bei der Osteotomie zu geben.

zur proximalen Tibia von medial. Die proximale Tibia wird freipräpariert und die Landmarks (proximaler Bereich der Tub. tibiae, kaudaler Gelenkspalt) werden definiert. Um die lateral liegenden Gefäße und Nerven vor dem Sägeblatt bei der Osteotomie zu schützen, wird die Muskulatur vorsichtig gelöst und der Bereich mit feuchten Tupfern gepolstert.

Bevor die Osteotomie erfolgt, wird der sog. TPLO-Jig, der aus einem speziellen Montagegestell besteht, über 2 Kirschner-Bohrdrähte am Tibiaplateau und der Tibiadiaphyse fixiert. Durch eine speziell am Jig fixierte Schablone (Sägelehre) kann der Sägeschnitt während des Eingriffs kontrolliert und angepasst werden (Abb. 12).

Weiterhin hat der Jig den Zweck, zu verhindern, dass eine iatrogene Varus- oder
Valgusfehlstellung oder eine Torsion der Tibia bei Gliedmaßen ohne Achsenabweichung entsteht. Ein weiterer Einsatzgrund ist, dass die oben dargestellten Fehlstellungen mithilfe dieser Fixierung im Rahmen der TPLO besser und genauer zu korrigieren sind $[11,15]$. Die Position des Jigs erfolgt an bestimmten Knochenpunkten der Tibia. Der proximale Bohrdraht wird direkt kaudal des medialen Seitenbands (MCL) etwa 3-4 mm unterhalb der Gelenkfläche eingebracht, wobei der Winkel zur Tibaachse etwa $90^{\circ}$ betragen sollte (Abb. 13).

Der distale Bohrdraht wird ebenfalls im $90^{\circ}$-Winkel in die distale Tibiadiaphyse gebohrt, sodass die über beide Gelenke zu justierenden Winkel des Jigs ebenfalls etwas unter $90^{\circ}$ zueinander stehen. Nach Ausrichtung des Jigs wird die Sägeschablone an den Jig fixiert (Abb. 14). Die Wahl der Größe des Sägeblatts wurde zuvor 


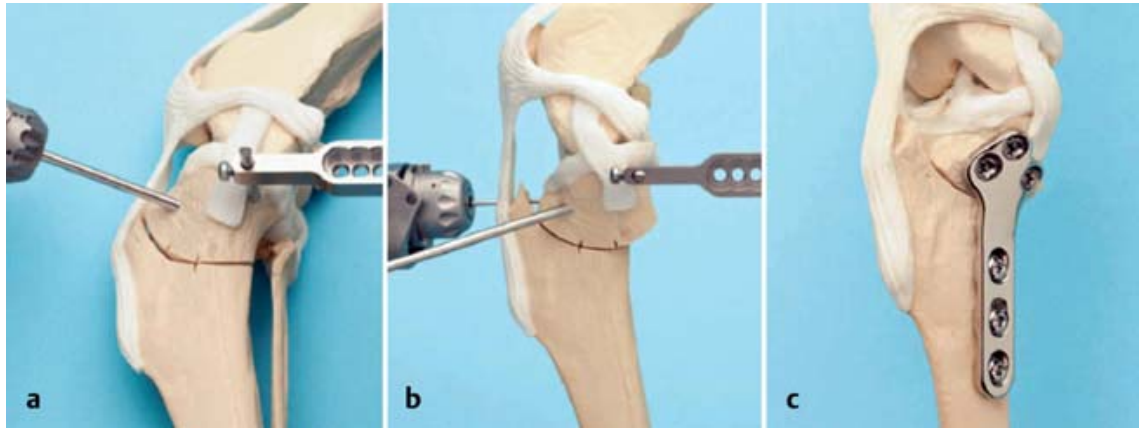

Abb. 15a bis c Nach der Osteotomie wird zur Erleichterung der Rotation des Tibiaplateaus ein Kirschner-Bohrdraht in das proximale Fragment eingebracht (a). Nach entsprechender Rotation des Tibiaplateaus wird es in der korrekten Position mithilfe eines weiteren Kirschner-Bohrdrahts fixiert (b), anschließend mit der TPLO-Platte fixiert (c).

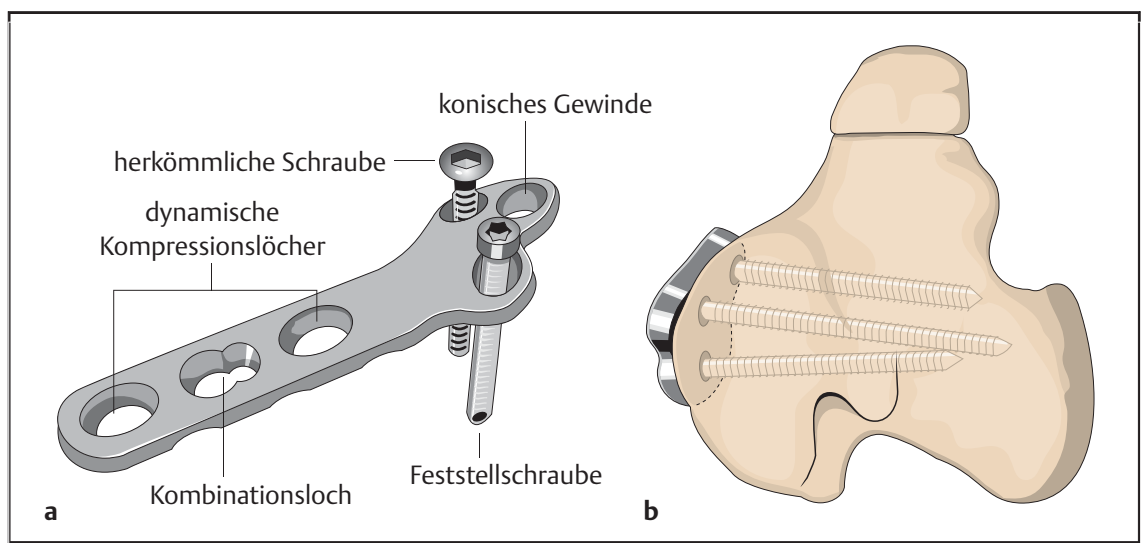

Abb. 16a und b Bei bestimmten winkelstabilen TPLO-Platten (hier z.B. der Fa. Synthes) ist durch die spezielle Biegung der Platte (a) und die vorgegebene Richtung der Schraubenlöcher (a) die Positionierung der Schrauben vorgegeben (b), sodass nur durch eine falsche Anbringung der TPLO-Platte die Möglichkeit besteht, dass die Schrauben das Kniegelenk penetrieren.
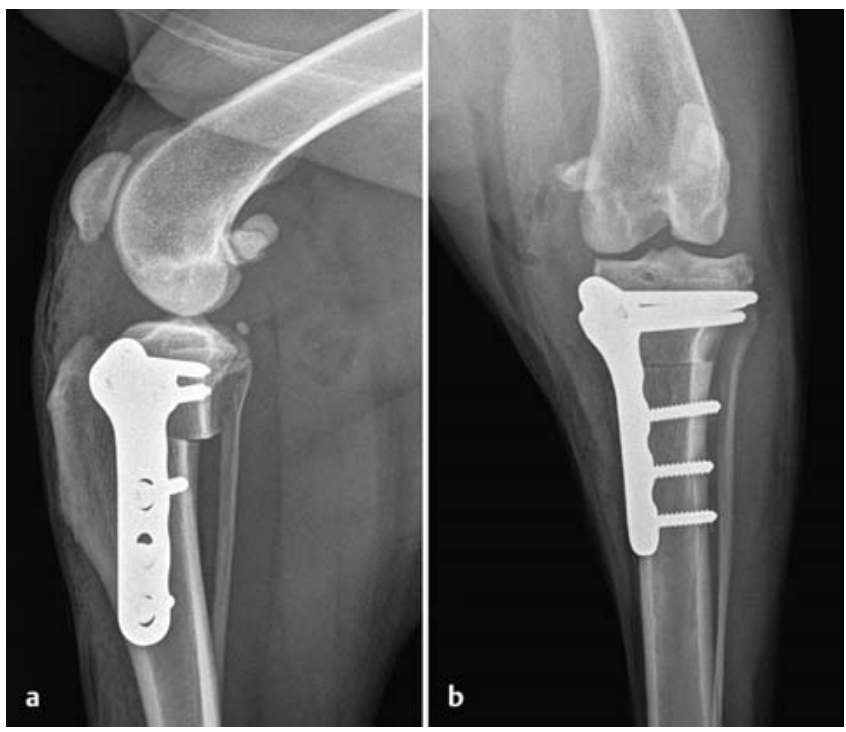

Abb. 17a und b Postoperative Röntgenaufnahmen im mediolateralen (a) und kraniokaudalen (b) Strahlengang zur Kontrolle der Position des Sägeschnitts, der Rotation des Tibiaplateaus, des Sitzes der Platte und der Schrauben und der durchgeführten Achsenkorrektur.

anhand der Röntgenbilder ausgewählt. Die ebenfalls am Röntgenbild bestimmten Distanzen D1 und D2 werden mithilfe einer Messschablone oder eines Kalipers auf den Knochen übertragen, wobei die Sägelehre an diesen beiden Punkten ausgerichtet wird.

Anschließend erfolgt die zirkuläre Osteotomie mit einer Schnittführung von medial nach lateral.
Um eine ideale Rotation des Tibiaplateaus durchführen zu können, muss der Osteotomieschnitt parallel zum Tibiaplateau und senkrecht zur sagittalen Ebene der Tibia in kraniokaudaler Richtung erfolgen [12].

Ist also die Position des Sägeschnitts festgelegt, wird mit der Osteotomie begonnen. Nach Durchtrennung der 1. Kortikalis erfolgt dann an der Osteotomielinie das Setzen der Markierungen für das individuelle Rotieren des Tibiaplateaus („Leveln“). Das Ausmaß der Rotation wurde zuvor anhand der gemessenen individuellen Steilheit des TPA und der Größe des Sägeblatts aus der Tabelle abgelesen (Tab. 1). Die Markierungen werden im Knochen mittels Schablone oder Kaliper zunächst an der oberen Schnittlinie mithilfe eines feinen Meißels angelegt (Abb. 16) und anschließend der zu rotierende Abstand auf der unteren Schnittkante der Tibia markiert. Danach wird die Osteotomie vervollständigt. Zur Erleichterung der Rotation wird ein weiterer Kirschner-Bohrdraht von medial in das proximale Fragment in proximodistale Richtung eingebracht (Abb. 15). Vor der Rotation werden die Wundtupfer entfernt. Nach entsprechender Rotation des Tibiaplateaus wird es in der korrekten Position mithilfe eines weiteren Kirschner-Bohrdrahts von kranial aus vorfixiert und anschließend mit der für den Patienten passende TPLO-Platte fixiert (Abb. 16). Bei verschiedenen Systemen muss dabei die Platte noch dem Knochen angepasst werden, wobei von einigen Firmen die Platte bereits in vorgebogenem Zustand jeweils für die rechte und linke Tibia ausgeliefert wird.

Im Zuge der Korrektur des Tibiaplateauwinkels können wie oben beschrieben durch zusätzliche Sägeschnitte noch weitere Korrekturen von Achsen- oder Rotationsfehlstellungen der proximalen Tibia vorgenommen werden.

\section{Intra- und postoperative Kontrolle} der TPLO

Die Kontrolle der korrekten Schraubenposition kann bereits intra operationem mithilfe eines C-Bogens fluoroskopisch vorgenommen werden.

Eine Fehlpositionierung von Schrauben ist einer der häufigsten Fehler bei der TPLO in Verbindung mit der Verwendung von nicht winkelstabilen Platten. 
Häufig befinden sich die Schrauben im Gelenk, sodass sie entweder neu positioniert werden oder im Falle zu langer Schrauben durch entsprechend kürzere ausgewechselt werden müssen. Bei den winkelstabilen Platten (z.B. Fa. Synthes) ist die Richtung der Schrauben bereits vorgegeben (Abb. 16). Die Komplikation der Fehlpositionierung ist daher nur bei falsch positionierter Platte möglich.

Eine weitere Kontrolle erfolgt nach dem routinemäßigen Wundverschluss. Es werden zur Überprüfung der Korrektur durch die TPLO Röntgenaufnahmen im mediolateralen und kraniokaudalen Strahlengang angefertigt, an denen der Sitz der Platte und der Schrauben, die korrekte Position des Sägeschnitts und die durchgeführte Korrektur des Tibiaplateaus sowie der Achse der Tibia kontrolliert werden (Abb.17). Die durchgeführte Rotation des Tibiaplateaus kann im kaudalen Bereich nochmals überprüft werden. Im weiteren Verlauf sollten röntgenologische Kontrollen des Kniegelenks erfolgen, um den Heilungsverlauf und die Durchbauung des Osteotomiespalts verfolgen zu können. Die Platte wird in der Regel belassen.

\section{Literatur}

${ }^{1}$ Fehr M. Ätiologie, Pathogenese, Diagnose und Therapie von Hunden mit einer Ruptur des kranialen Kreuzbandes. Kleintierprax 2007; 52: $374-382$

2 Henderson RA, Milton JL. The tibial compression mechanism: a diagnostic aid in stifle injuries. J Am Anim Hosp Assoc 1978; 14: 474479

${ }^{3}$ Robins G. Canine Orthopedics. Philadelphia: Verlag Lea \& Febiger; 1990: 693-705

${ }^{4}$ Morris E, Lipowitz AJ. Comparison of tibial plateau angles in dogs with and without cranial cruciate ligament injuries. J Am Vet Med Assoc 2001; 218: 363-366

5 Paley D, Pfeil J. Principles of deformity correction around the knee. Orthopäde 2000; 29 : 18-38

6 Dismukes DI, Tomlinson JL, Fox DB et al. Radiographic measurement of the proximal and distal mechanical joint angles in the canine tibia. Vet Surg 2007; 36: 699-704

7 Jerram RM, Walker AM. Cranial cruciate ligament injury in the dog: pathophysiology, diagnosis and treatment. New Zeal Vet J 2003; 51: $149-158$

8 Morris E, Lipowitz AJ. Comparison of tibial plateau angles in dogs with and without cranial cruciate ligament injuries. J Am Vet Med Assoc 2001; 218: 363-366

${ }^{9}$ Warzee CC, Dejardin LM, Arnoczky SP. Effect of tibial plateau leveling on cranial and caudal tibial thrusts in canine cranial cruciate-deficient stifles: an invitro experimental study. Vet Surg 2001; 30: 278-286

10 Moeller EM, Cross AR, Rapoff AJ. Change in tibial plateau angle after tibial plateau leveling osteotomy in dogs. Vet Surg 2006; 35: 460464
${ }^{11}$ Dejardin LM. Tibial Plateau Leveling Osteotomy. In: Slatter D, ed. Textbook of Small Animal Surgery. 2nd ed. Philadelphia: Saunders; 2003: 2133-2143

12 Slocum B, Slocum TD. Tibial plateau leveling osteotomy for repair of cranial cruciate ligament rupture in the canine. Vet Clin North Am Small Anim Pract 1993; 23: 777-795

13 Reif $U$, Hulse DA, Hauptmann JG. Effect of tibial plateau leveling on stability of the canine cranial cruciate-deficient stifle joint: an in vitro study. Vet Surg 2002; 31: 147-154

${ }^{14}$ Shahar R, Milgram J. Biomechanics of tibial plateau leveling of the canine cruciate-deficient stifle joint: a theoretical model. Vet Surg 2006; 35: 144-149

15 Palmer RH. Tibial plateau leveling osteotomy. Arlington, VA: Proc. of the 10th American College of Veterinary Surgeons Symposium; 2000: 271-275

Prof. Dr. med. vet.

\section{Andrea Meyer-Lindenberg}

Klinikleitung

Vorstand der Chirurgischen und Gynäkologischen Kleintierklinik

Chirurgische und Gynäkologische Kleintierklinik Ludwig-Maximilians-Universität München

Veterinärstraße 13 80539 München

meyer-lindenberg@chir.vetmed.unimuenchen.de 